\title{
Préface : Le Réseau international sur la professionnalisation des communicateurs
}

\author{
Patrice de la Broise, Maître de conférences HdR, \\ Université Charles-de-Gaulle-Lille 3, GERIICO, \\ patrice.delabroise@univ-lille3.fr \\ Vincent Brulois, directeur de I'UF Communication, \\ Université Paris 13, PRES Sorbonne Paris Cité, LabSIC, \\ brulois@sic.univ-paris13.fr
}

Nous savons l'utilité des acronymes et en connaissons les limites. RESIPROC Réseau international sur la professionnalisation des communicateurs - n'est guère respectueux de l'orthographe, mais il dit bien la philosophie d'un réseau associant étroitement des professionnels de la communication de deux mondes complémentaires, celui de l'entreprise et celui de l'enseignement et de la recherche, dans un projet commun d'analyse et d'accompagnement d'une professionnalisation aux métiers et fonctions de la communication.

En mai 2010, à l'occasion du 78e Congrès de l'Acfas ${ }^{1}$ à Montréal, plusieurs chercheurs et praticiens s'interrogeaient déjà sur la reconnaissance professionnelle de la fonction Communication et de ses métiers dans le cadre d'un colloque: Communication sociale et publique : prisme ou foyer? De ce côté de l'Atlantique, des collègues québécois - emmenés par Marc D. David (Université de Sherbrooke) et Bernard Motulsky (UQAM) - lançaient une grande enquête sur les pratiques en communication visant à caractériser cette fonction et son périmètre de missions dans les institutions publiques et privées au Canada. De l'autre côté, en France et en Belgique, plusieurs universitaires - dont nous étions - travaillaient également à une sociologie des groupes professionnels en communication, notamment sur les segments de la communication interne et de la communication publique. La

\footnotetext{
${ }^{1}$ Association francophone pour le savoir, créée au Canada, en 1923, sous le nom d'Association Canadienne-Française pour l'Avancement des Sciences (ou ACFAS). Elle organise un Congrès, toujours au Québec, depuis 1932.
} 
rencontre était alors d'évidence, les uns souhaitant discuter et prolonger leurs résultats ou leurs intuitions avec les autres. Un groupement d'universitaires et de praticiens s'est constitué à la faveur d'une démarche collective, surtout francophone à l'origine, d'étude de la fonction Communication et des parcours de formation et d'emploi en communication.

Afin de ne pas laisser passer l'idée, l'occasion était trop belle de se retrouver l'année suivante, dans le même cadre, autour d'un colloque $a d$ hoc où la discussion pouvait s'engager. En mai 2011, Marc D. David encore et Dany Baillargeon, recevant le $79^{\mathrm{e}}$ Congrès de l'Acfas en leur Université de Sherbrooke, organisèrent ainsi le colloque 434 sur le thème des perspectives de développement et des pratiques en communication dans leurs modes d'organisation et leurs enjeux de formation. Une vingtaine de chercheurs et des praticiens prirent part à l'événement, partageant leurs travaux et leurs réflexions, élargissant le cercle de professionnels des parties prenantes d'un intérêt pour la thématique, commençant à dessiner un programme d'étude et de recherche. En marge de ce colloque, une réunion entre les différents acteurs a donné corps à ce programme et au RESIPROC : partager et fédérer nos regards sur les métiers, fonctions, pratiques, territoire d'action des professionnels de la communication, ainsi que sur leurs instances de représentation ou les formations en communication.

Trois constats ont été posés. Quelles que soient nos différences culturelles - liées à la géographie (Belgique, Canada, France), à notre environnement professionnel (entreprise, institution publique, université), à notre rôle (praticien, enseignant, consultant, chercheur), à notre angle d'approche (communication, communication interne, marketing, relations publiques) -, nous partageons un intérêt commun à structurer nos réflexions et nos travaux. Il s'agit pour les uns de faire reconnaître un métier voire de légitimer une profession (référentiel Métiers); pour d'autres de s'intéresser aux parcours des communicants et aux formations en communication (référentiel Formations) jusque, et y compris, dans leurs fondements scientifiques (les sciences de l'information et de la communication). In fine, il s'agit pour tous d'interroger la professionnalisation des communicateurs - version québécoise - ou communicants - version française -, à partir du monde de l'université comme du monde de l'entreprise, en tout cas avec ceux-là mêmes qui font métier de communiquer. Pour cela (deuxième constat), on ne peut se passer d'identifier aussi précisément que possible l'organisation de ces métiers de la communication en associations ou en syndicats qui fédèrent les professionnels, qui déterminent un territoire d'actions, qui identifient des métiers et qui mettent à jour des pratiques. Cette nécessaire identification doit donc donner lieu à une cartographie précise, sinon exhaustive, des organisations professionnelles. Celle-ci est à mettre en parallèle avec une cartographie similaire des formations universitaires. L'une et l'autre, doivent permettre de détecter les recouvrements, les évolutions, les jeux d'acteurs, en bref de comparer les référentiels Métiers et Formations. Enfin 
(troisième constat), cette connaissance documentée ne peut répondre aux attentes de tous que si elle est partagée. Il s'agit donc de mettre en place un réseau d'échange, de discussion et de dialogue autour de cette question de la professionnalisation.

C'est donc pour identifier, structurer et partager que le Réseau international sur la professionnalisation des communicateurs a été constitué, afin d'établir une cartographie des associations, de dresser un portrait des métiers de la communication, de clarifier les profils et les trajectoires professionnelles, de comprendre les évolutions des pratiques, d'interroger le rôle des formations universitaires en communication, de renforcer le dialogue entre les communautés professionnelles et universitaires, en fin de compte, de définir ce qu'il faut entendre par professionnalisation en et de la communication.

À ces objectifs, il convenait d'en ajouter un autre : rythmer, sans quoi le réseau avait toutes les chances de rester informel... C'est pourquoi un premier programme de travail a été entrepris, encore empirique et certainement incomplet. D'abord exploratoire, notre entreprise s'est enrichie d'un protocole d'enquête qualitative visant à mieux connaître la structuration associative des métiers de la communication, à cartographier et radiographier cette représentation professionnelle en divers segments et selon différentes logiques culturelles et territoriales. La collecte, organisée par binômes de chercheurs, concernait, d'une part, les productions sémio-discursives et déontiques (chartes, référentiels, codes de déontologie) des associations professionnelles en communication et, d'autre part, les récits biographiques et les discours de leurs principaux représentants. Quatre segments professionnels ont ainsi été retenus : la communication sociale et interne, la communication publique et territoriale, les relations publiques (incluant les relations Presse) élargies à la communication dite «corporate » (en France et Belgique), dite affaires publiques ou institutionnelles, et enfin la communication commerciale ou communication marketing. Cette analyse des dynamiques de professionnalisation à travers les instances représentatives a été présentée à Roubaix en mars $2012^{2}$ (Lépine et al., 2012), lors d'une session dédiée aux programmes de recherche collective du colloque international sur la question des normes, coorganisé par l'Ica ${ }^{3}$, la Sfsic ${ }^{4}$ et le laboratoire GERIICO ${ }^{5}$ (Université Lille3).

\footnotetext{
${ }^{2}$ Lépine, V., Brulois, V., Lambotte, F., Coyette, C., David, M. D., Baillargeon, D., Chauvin, D. (2012, 9 mars). De la reconnaissance mutuelle et publique des professionnels de la communication. Actes du colloque Communiquer dans un monde de normes, Roubaix.

${ }^{3}$ International Communication Association, fondée aux Etats-Unis, en 1950, sous le nom de National Society for the Study of Communication (NSSC).

${ }^{4}$ Société Française des Sciences de l'Information et de la Communication, créée en France, en 1970, avant même que la discipline ne soit institutionnalisée.

${ }^{5}$ Groupement d'Étude et de Recherche Interdisciplinaires en Information et Communication (EA 4073)
} 
En suivant, au moins deux étapes sont en cours. D'une part, une enquête sur l'identité professionnelle du communicant interne afin de décrypter les évolutions et les aspirations des acteurs de cette fonction en réaménagement permanent. Diligentée par l'Afci $^{6}$ et Inergie sur proposition de Vincent Brulois (Université Paris 13), cette enquête se propose d'aller à la rencontre de responsables ou directeurs(trices) de communication interne dans leur diversité et de les interroger sur leur parcours (de formation et d'emploi), leur place dans l'entreprise (factuelle et symbolique), leurs pratiques et leurs perspectives. Une première synthèse en sera donnée fin juin 2013. D'autre part, un nouveau colloque organisé en mai 2013 à Québec, à l'initiative de Valérie Lépine (Université Grenoble 2 et responsable de la commission Formation de la Sfsic) et Marc D. David (Université de Sherbrooke). Toujours dans le cadre du $81^{\mathrm{e}}$ Congrès de l'Acfas, il sera question d'analyser les dispositifs d'apprentissage et de formation des communicateurs. Entre autres questionnements, en dépit de spécificités sectorielles ou techniques, quelles lectures et quelles structurations en termes de dispositifs de formation peut-on faire des acquisitions ou des postures théoriques, méthodologiques et pratiques qui contribuent au professionnalisme des acteurs responsables des communications organisationnelles?

Une dynamique semble donc enclenchée, dont la rencontre inaugurale de 2011 a fait office de starter. Elle a pour caractéristique de créer des ponts entre les mondes de l'université et de l'entreprise, de générer une conversation constructive entre professionnels des différents processus menant à la professionnalisation. À travers ce prisme, il s'agit tout à la fois de clarifier les métiers de la communication, les organisations représentatives, les formations, que de s'intéresser aux individus (profil, trajectoire professionnelle, vision, attentes); de questionner les normes en communication (qu'est-ce qu'une communication réussie et sur quels critères se construit son évaluation ?) que de percevoir la façon dont les formes sont bousculées sous la poussée de constantes innovations techniques (la mode n'est-elle pas aujourd'hui aux réseaux sociaux?); de constituer un corpus de savoirs que d'identifier les savoir-faire et savoir-être et de comprendre leurs évolutions à l'aune des transformations de l'entreprise. Cette dynamique a pour fondement la conviction que nos connaissances sur les acteurs spécialisés, les métiers, les processus de professionnalisation et d'institutionnalisation des fonctions ainsi que l'interrogation réflexive quant à nos pratiques d'ingénierie pédagogique doivent s'actualiser dans une démarche collective, internationale et réciproque.

\footnotetext{
${ }^{6}$ Association Française de Communication Interne, fondée en 1989. Seule association, en France, qui a pour objet spécifique la communication interne.
} 